\title{
Dielectric Behavior of Medical PMMA Polymer Filled With Copper Nanobud Particles
}

\author{
Ismail Ibrahim Marhoon*1, Amer Hameed Majeed², Mohammed Ali Abdulrehman ${ }^{3}$ \\ 1,2,3 Materials Engineering Department / Mustansiriyah University, Baghdad, Iraq \\ *E-mail: isibmr@uomustansiriyah.edu.iq
}

\begin{abstract}
In this paper, the influence of weight fraction and frequency on dielectric features of polymethylmethacrylate filled with Copper nanobud particles has been investigated. Copper nanoparticles (Cu-NPs) have been synthesized by chemical reduction method. The resultant Copper nanoparticles were characterized by UV-Vis spectroscopy and X-ray diffraction (XRD). Results show that the Cu-NPs have bud shapes, and their average particle size obtained from the XRD study is $53.78 \mathrm{~nm}$. For the resultant composite materials, the dielectric behavior of composite materials reinforced with $5 \%, 10 \%$, $15 \%$, and $20 \%$ weight fractions of Copper nanobud particles and frequency ranges of $50,250,10^{3}, 10^{4}, 10^{5}$, and $10^{6} \mathrm{~Hz}$ at $25{ }^{\circ} \mathrm{C}$ were investigated. Results reveal that the dielectric constant, dielectric loss factor and dissipation factor increased with the increase in weight fraction of Copper nanobud particles as a result of their high conductivity. The dielectric constant, dielectric loss and dissipation factor have decreased with the frequency increase. Such dielectric parametric investigations are useful to understand their application limitations not only for electronic apllications but also for feasible medical uses.
\end{abstract}

\section{Keywords}

Nano-Copper particles, nanobuds, weight fractions, frequency, dielectric properties, composite materials, PMMA

\section{Introduction}

Conductive polymer composites are lightweight materials and they can combine the characteristics of electrically conductive metals with polymer capability. These composites are used in various applications such as acoustic emission sensors, angular acceleration accelerometers, integrated decoupling capacitors, electronic packaging, electromagnetic frequency interference shields, and antistatic devices [1-4]. The mixture of various fillers and fibers with polymer matrices can produce different composite materials with significant dielectric properties for electronic application [57]. Conductive polymer composites recently received increased attention due to their interesting electrical and electronic properties. One type of these composite materials is particulate-filled polymer matrix conductive composite, which comprises a polymer matrix with a second dispersed phase that is typically either a few non-metallic elements such as carbon or metal-based fillers (micro and nano) [8-11]. The actual use of filled polymers strongly depends on the capability of the filler particles to homogeneously disperse through the matrix [12]. The interface features between the polymer matrix and filler particles also intensely influence the performance and characteristics of these conductive composites [13]. One of the main features of filled composite materials is that their dielectric properties can be extensively altered by selecting the conductivity of filled components and the size and shape of the polymeric matrix [14]. Alumina, titania, Copper oxide, lithium fluoride, nickel salt, graphite, aluminum, and lead titanate have been commonly used in to fill various polymer matrices. Thus, composites with enhanced electrical property responses have been synthesized [15-22].

The efficiency of Copper as an active agent with low toxicity is mainly relevant in the antibacterial treatment and has been documented for a long time [23, 24]. Copper is still a desirable applicant for future conducting materials due to its low cost and abundance. However, recent studies demonstrate that Copper nanoparticles are oxidized from the surface under conditions of room temperature $[25,26]$. The problems of oxidation and aggregation can be avoided using numerous protecting agents, such as polymers and organic ligands $[27,28]$.

Polymethylmethacrylate (PMMA) can be used for medical applications since they are physiologically harmless components and they are not attacked by molds and enzymes due to its low capacity for water absorption. PMMA has a high degree of compatibility with humanoid tissue. For, instance, it can be used in the production of rigid intraocular lenses that are implanted in the eye at for cataracts treatment. PMMA polymer materials are utilized due to their rigidity and strength to produce sensors, electromagnetic frequency interference shields, medical transplant components and antistatic devices. The addition of metal powders to PMMA polymer increases its electrical and thermal conductivity [23, 24]. 
Dielectric studies are useful to understand physical state of biological water, proton transport, and the difference between normal and tumorous tissue. Knowledge of the dielectric properties under specified frequencies is very important for medicinal applications in diagnostic and therapeutic medicine [29, 30].

Accordingly, this study deals with the effect of the nanobudCopper additive and the frequency of alternating current on dielectric properties of PMMA composite. The dielectric properties of PMMA polymer nanobud-Copper composite materials have been studied for practical medical applications at frequencies below $1 \mathrm{MHz}$.

\section{Materials and Methods}

\subsection{Chemicals and materials}

L-ascorbic acid (CDH Chemicals, purity: 99\%) and isonicotinylhydrazide (INH) were provided by Sandoz Corporation. On the other hand, Copper (II) sulfate pentahydrate (purity: 99\%) and sodium hydroxide (purity: 97.5\%) were supplied by Thomas Baker Corporation. Polyvinylpyrrolidone K30 (Ourchem Sinopharm Chemical Reagent, purity: 98\%-99\%) and all chemicals were used as supplementary without purification. The chemical reactions of these materials were carried out by using distilled water from our lab. Polymer components of PMMA, which comprise PMMA particles, were provided by VERACRIL Corporation. PMMA has a particle size of $75 \mathrm{~mm}$, a density of $1.25 \mathrm{~g} / \mathrm{cm}^{3}$, and purity of $90 \%-99 \%$. The initiated methacrylate monomer (IMM) self-curing base resin was provided by Duracryl Plus and manufactured by Spofa Dental.

\subsection{Synthesis of Bud-Shaped Cu-NPs}

The Cu-NPs were synthesized by the chemical reduction method [23] using precursor Copper sulfate pentahydrate, as shown in Figure 1. In the usual procedure, the aqueous solution of $\mathrm{CuSO}_{4} .5 \mathrm{H}_{2} \mathrm{O}$ was prepared by dissolving $0.25 \mathrm{gm}$ in $1 \mathrm{~L}$ deionized water and was added to a polyvinylpyrrolidone (PVP) $\left(\mathrm{C}_{6} \mathrm{H}_{9} \mathrm{NO}\right)_{\mathrm{n}} \mathrm{K}-30$ solution of 10 $\mathrm{gm} / \mathrm{L}$ to provide an excellent covering agent to prevent precipitation and agglomeration of the particles. PVP has been frequently employed in the chemical synthesis of metal nanoparticles as stabilizers. Then, the solution of $20 \mathrm{gm} / \mathrm{L}$ $\mathrm{NaOH}$ was added drop by drop to adjust the $\mathrm{pH}$ of the solution to 7. The reaction mixture was continuously stirred for 60 minutes. L-Ascorbic Acid $\left(\mathrm{C}_{6} \mathrm{H}_{8} \mathrm{O}_{6}\right) 0.034 \mathrm{M}$, which is an antioxidant and a mild reducing agent, was prepared by dissolving $6 \mathrm{~g}$ of L-ascorbic acid in $1 \mathrm{~L}$ deionized water and stirring at $25^{\circ} \mathrm{C}$ temperature for 60 minutes. The mixture was heated and maintained at $65^{\circ} \mathrm{C}$ for approximately 15 minutes. The ascorbic acid was added as a drop to the reaction mixture. The color of the solution changed to light yellow as shown in Figure 2a, thereby indicating the seed formation of nanoCopper. Then, the isonicotinylhydrazide $\left(\mathrm{C}_{6} \mathrm{H}_{7} \mathrm{~N}_{3} \mathrm{O}\right)$ solution with a concentration of $137.14 \mathrm{mg} / \mathrm{L}$ was slowly added to the solution. The color of the reaction mixture was transformed to reddish brown as shown in Figure $2 b$, thus signifying the formation of Copper nanobuds. Heating and stirring were maintained for $60 \mathrm{~min}$ to promote the growth of nanobuds. The resultant solution was then centrifuged for 15 minutes. Finally, the product was washed with ethanol and dried in air.

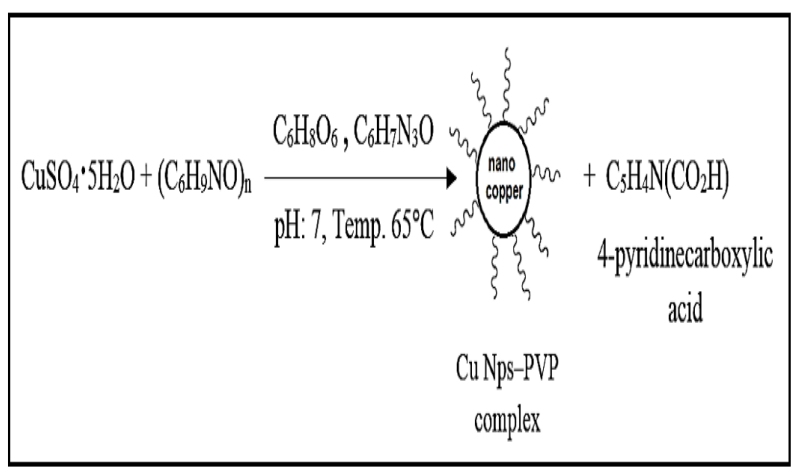

Figure 1: Illustration of synthesis of Bud-Shaped Cu-NPs. Copper sulfate reacts with PVP to form a complex that is reduced by $\mathrm{L}$-ascorbic acid and isoniazid consequent in 4-pyridinecarboxylic acid $\mathrm{C}_{5} \mathrm{H}_{4} \mathrm{~N}\left(\mathrm{CO}_{2} \mathrm{H}\right)$ and BudShaped $\mathrm{Cu}-\mathrm{NPs}$.

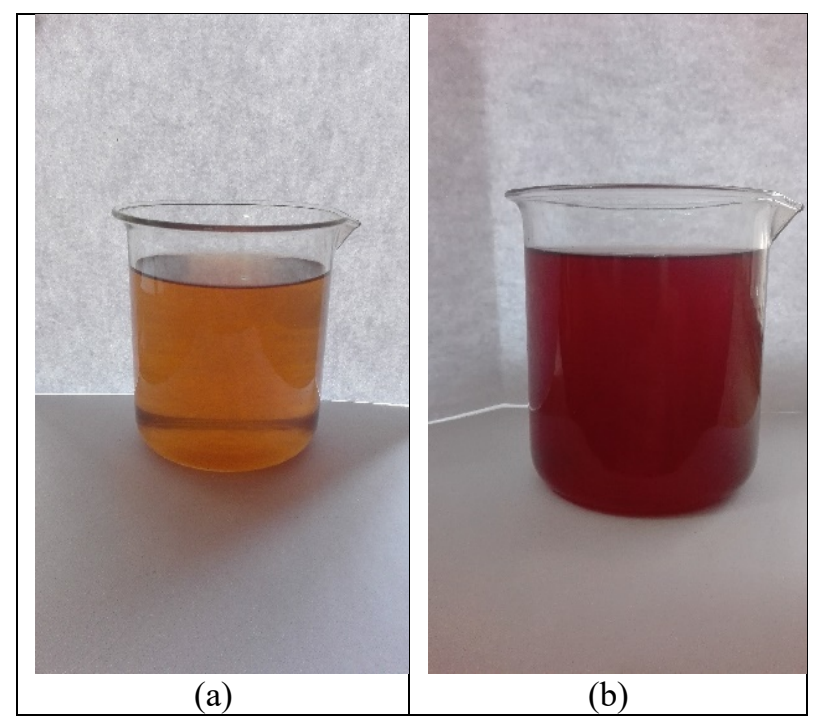

Figure 2: Different steps of Copper Nano-buds particle synthesis solution, (a) light yellow solution after addition L-ascorbic acid; (b) reddish brown solution after addition INH to the previous solution

\subsection{Composite preparation}

PMMA materials were used in the present work as matrix and the Copper nanobuds as a filler particle to attain a weight amount of Copper nanobuds and PMMA polymer powder by using electronic balanced with an accuracy of four digits. The weight percentages of Copper nanobuds are 0 wt. $\%, 5$ wt. $\%, 10$ wt. $\%, 15$ wt. $\%$, and 20 wt. $\%$. The 
$\mathrm{PMMA}+\mathrm{Cu}$ nanobud composite powder mixtures are combined by hand, and the homogenizing of powder mixture was tested by microscopic examination. The composite powder was then placed in a disc shape mold with a diameter of approximately $23 \mathrm{~mm}$ and a thickness of about $4.6 \mathrm{~mm}$. The IMM is added to (PMMA+ $\mathrm{Cu}$ nanobuds) the composite powder mixture by $2: 1$ volume ratio. The mixture of different weight percentages of $\mathrm{Cu}$ NPs was then compacted at a temperature of $100^{\circ} \mathrm{C}$ under a pressure of $10 \mathrm{MPa}$ for approximately 10 minutes to obtain coherent final disc sample shape. Then, it was cooled at $25^{\circ} \mathrm{C}$ and released from the mold as shown in Figure 3. The coating unit, Cressington sputter coater (108 autos), was used for deposition of thin film gold electrode on both sides of each sample to improve the electrical properties of the sample surface.

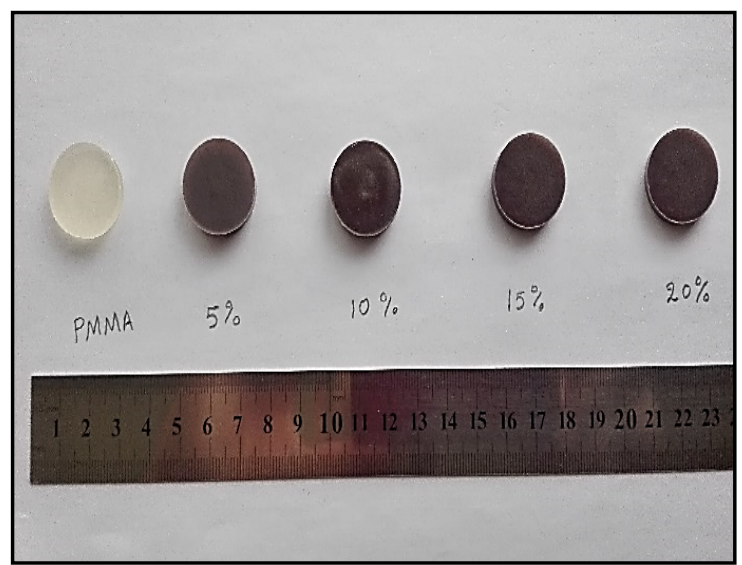

Figure 3: Dielectric test specimens at a different weight fraction

\subsection{Characterization}

The following studies have characterized the Copper nanoparticles. The UV-Vis spectra were recorded on a GENESYS UV-Vis spectrophotometer. UV-Vis stands for absorption or reflectance spectroscopy in ultravioletvisible spectral region. It uses light in the visible and adjacent ranges. The absorption or reflectance in the visible range directly affects the perceived color of the involved materials.

Atoms and molecules undergo electronic transitions in this region of the electromagnetic spectrum. Absorption spectroscopy is corresponding to fluorescence spectroscopy, in that fluorescence is related with transitions from the excited state to the ground state, whereas absorption processes transitions from the ground state to the excited state.

Powder X-ray diffraction (XRD) analysis was carried out on a Shimadzu labx-XRD 6000 unit with a $\mathrm{Cu}-\mathrm{K} \alpha$ radiation at a scanning speed of $8 \% \mathrm{~min}$, a sampling pitch of $0.02^{\circ}$, and a preset time of $0.15 \mathrm{~s}$. X-ray powder diffraction (XRD) stands for a speedy analytical method principally employed for phase detection of a crystalline material and can offer information on unit cell dimensions.

\subsection{Measurement of composite properties}

The Debye-Scherrer equation was used to estimate the size of crystallite as follows:

$$
D=\mathrm{K} \lambda / \beta \cos \theta
$$

Where $D$ is the average size of $\mathrm{Cu}-\mathrm{NPs}$, $\mathrm{K}$ : dimensionless shape factor 0.94 for spherical crystallites,

$\lambda$ : wavelength of $\mathrm{Cu} \mathrm{K}-\alpha 1\left(1.54056^{\circ} \mathrm{A}\right)$,

$\beta$ : full width at half maximum $(0.16590)$,

$\theta$ : diffraction angle.

The precision LCR meter device (Gwinstek LCR8000G) was used to measure the capacitive $\left(C_{P}\right)$, while the dissipation factor $(\tan \delta)$ and values were recorded from the electronic screen [31]. The dielectric constant $\left(\epsilon^{\prime}\right)$ and dielectric loss factors ( $\left.\epsilon^{\prime \prime}\right)$ can be obtained from these values using Equations 2 and 3, respectively. These measurement investigations for $50,250,10^{3}, 10^{4}, 10^{5}$, and $10^{6} \mathrm{~Hz}$ are at room temperature [32].

$$
\epsilon^{\prime}=\mathrm{C} \mathrm{L} / \epsilon_{\mathrm{o}} \mathrm{A}
$$

where $\epsilon^{\prime}$ is the dielectric constant, $\mathrm{L}$ is the thickness of the dielectric sample in $\mathrm{cm}, \mathrm{A}$ is the cross-section area in $\mathrm{cm}^{2}$, and $\epsilon_{\mathrm{o}}$ is the vacuum permittivity $\left(8.854 \times 10^{-14} \mathrm{~F} / \mathrm{cm}\right)$

$$
\epsilon^{\prime \prime}=\tan \delta \times \epsilon^{\prime}
$$

Where $\epsilon^{\prime \prime}$ is the dielectric loss factor, and tan $\delta$ is the dissipation factor.

\section{Results and discussion}

In the present research, $\mathrm{Cu}$ nanobuds have been prepared through a chemical reduction method using Copper sulfate pentahydrate as a precursor.

\subsection{UV-Vis Spectrum of Cu Nanobuds}

Figure 4 demonstrates the UV-Vis spectrum of $\mathrm{Cu}$ nanobuds. The characteristic plasmon absorption band for Copper nanobuds was observed at $574.8 \mathrm{~nm}$ indicated to undergo bathochromic with the size increase. For $\mathrm{Cu}$ NPs, the surface plasmon resonance band was found to be approximately $560-570 \mathrm{~nm}[33,34]$ and was indicated to undergo hypsochromic with size decrease [30]. In general, the hypsochromic and surface plasmon resonance is affected by the particle size distribution when the particles are spherical. Therefore, the band observed at $574.8 \mathrm{~nm}$ is suggested to be attributed to the Copper nanobuds. 


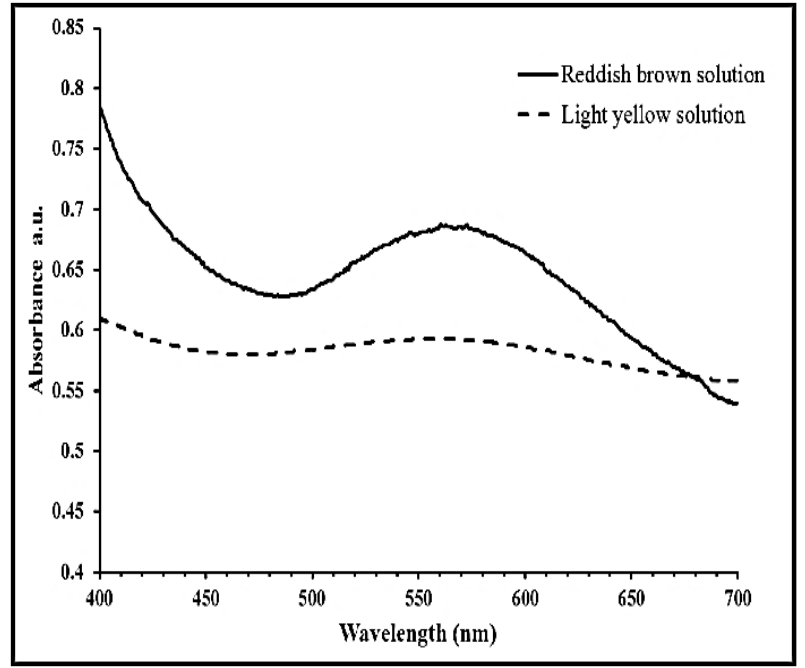

Figure 4: UV-Vis spectrum of $\mathrm{Cu}$ nanobuds particles synthesis solution, (a) light yellow solution after addition L-ascorbic acid; (b) reddish brown solution after addition INH to the previous solution at room temperature

\subsection{XRD of Cu Nanobuds}

In Figure 5, the XRD of $\mathrm{Cu}$ nanobuds exhibits a peak at $44.14^{\circ}$ and $76.87^{\circ}$, thereby matching planes $\{111\}$ and $\{220\}$ of the face-centered cubic structure of $\mathrm{Cu}-\mathrm{NPs}$. However, the $63.94^{\circ}\{220\}$ plane shows a trace of impurities of cuprite $\left(\mathrm{Cu}_{2} \mathrm{O}\right)$ and these resultant peaks are agree with Smapath et al. [23]. All possible $\mathrm{Cu}$ nanobud peaks are allocated by using match software in comparison with those defined in the PDF No. 00-0040836. Using Equation 1, the size of the crystallite is approximately $53.78 \mathrm{~nm}$, which may indicate a high surface area and the ratio of surface area to volume of the $\mathrm{Cu}$ nanobuds.

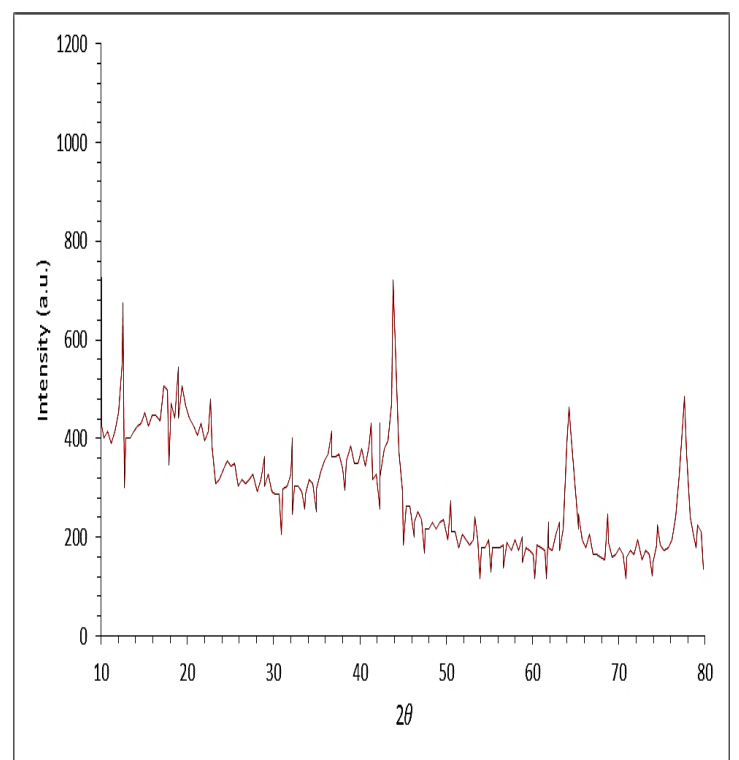

Figure 5: XRD pattern of $\mathrm{Cu}$ nanobuds

\subsection{Frequency Effect}

The effect of frequency and weight fraction on the dielectric constant $\epsilon^{\prime}$, dielectric loss factor $\epsilon^{\prime \prime}$, and dissipation factor $\tan \delta$ for all samples at room temperature is shown in Figures 6-8. The results reveal that the dielectric constant $\epsilon^{\prime}$, dielectric losses factor ( $\left.\epsilon^{\prime \prime}\right)$, and dissipation factor $(\tan \delta)$ are decreased with the increase in frequencies. Such a result can be related to the permittivity of dielectric materials, which depends on the movement of charges and dipoles. Therefore, when dielectric materials are subjected to an alternation of the electric field, the field direction changes. Furthermore, with each electric field reversal direction, the dipoles attempt to reorient with a new field direction in a process that requires some finite time. These interfacial dipoles have less time to orientate into the direction of the applied electric field that changes direction with time. The intensity of the alternating electrical field is represented by the frequency range of applied voltage, where the frequency affects the dipole alignment of the dielectric material. Therefore, dielectric relaxation is revealed to include orientation polarization. Figure 7 shows a dielectric loss as a function of the frequency of Copper nanobud composites. The figure shows that when the applied field has low frequency, the dielectric loss is high and decreases with increasing frequency. This decrease is related to the reduction in the charge polarization influence when the frequency increases. These findings are in agreement with reported results in $[30,35]$.

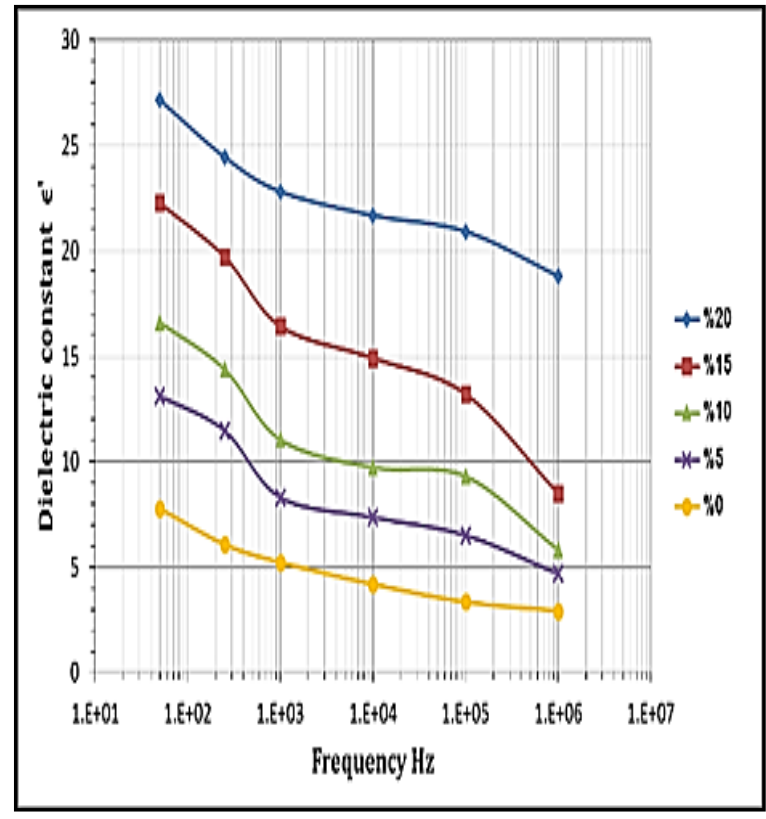

Figure 6: Variation of dielectric constant $\left(\epsilon^{\prime}\right)$ with log frequencies 


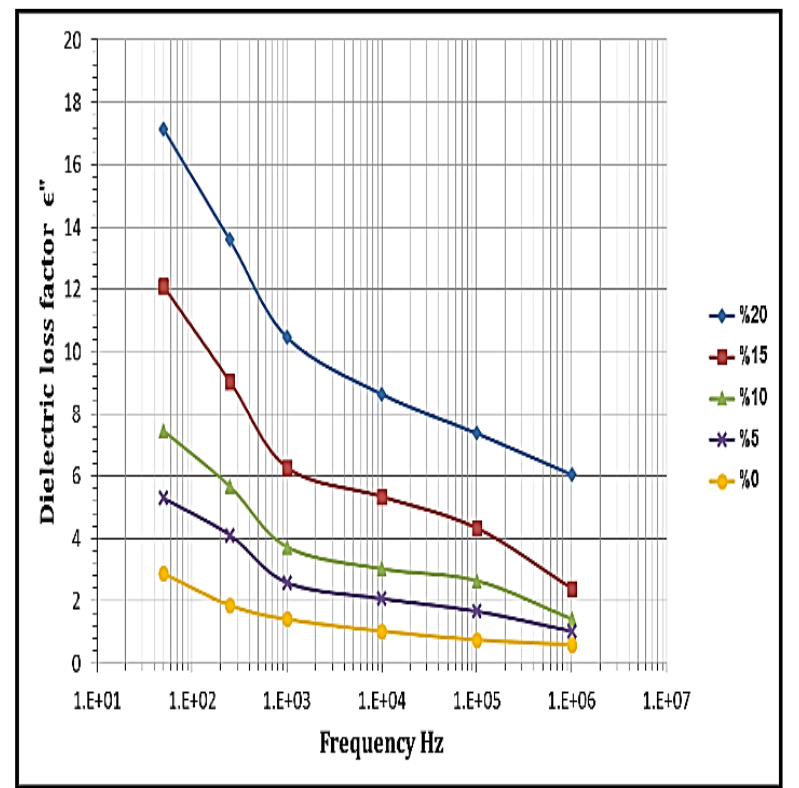

Figure 7: Variation of dielectric loss factor ( $\left.\epsilon^{\prime \prime}\right)$ with log frequencies

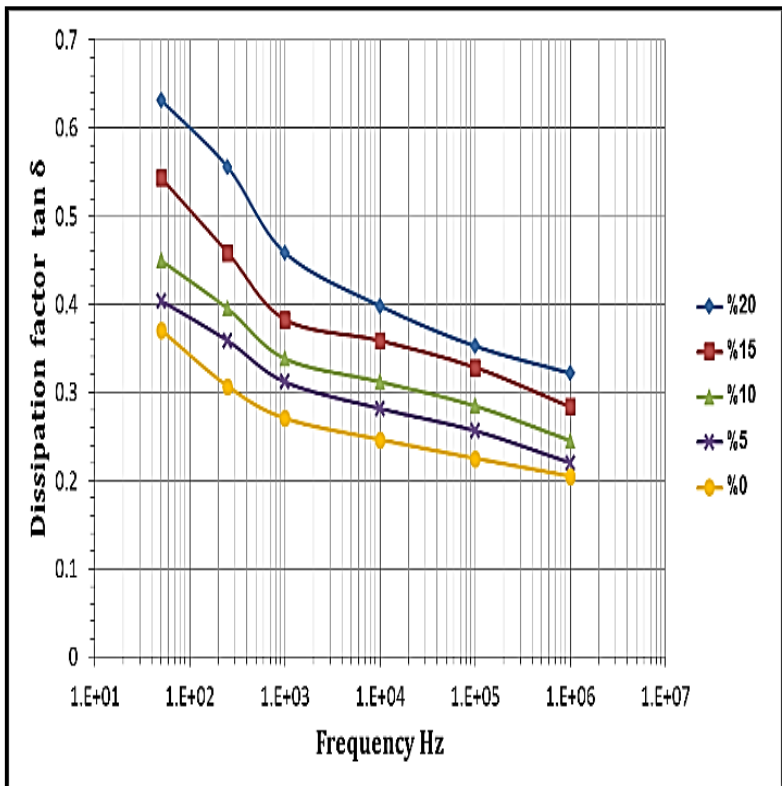

Figure 8: Variation of dissipation factor $(\tan \delta)$ with different frequencies.

\subsection{Weight Fraction Effect}

The effect of different weight fractions of Copper nanobuds was investigated in the present work. The results showed that weight fractions of Copper nanobuds affected the dielectric properties of the resultant composite materials. The dielectric properties for the samples of $0 \%, 5 \%, 10 \%, 15 \%$, and $20 \%$ are shown in Figures 6-8.

Compared to the electrical conductivity of Copper which is $5.98 \times 10^{7} \mathrm{~S} / \mathrm{cm}$, the dielectric constant of PMMA is $2.8-4$, and its electrical conductivity is $10^{-14}-10^{-15} \mathrm{~S} / \mathrm{cm}$. Therefore, increasing the weight fraction of Copper nanobuds at constant frequency results in the increase in dielectric constant. The maximum value of dielectric losses and dielectric constant reached 17.14 and 27.15, respectively at $20 \mathrm{wt} \%$ with $50 \mathrm{~Hz}$. The increase in dielectric constant and dissipation factor values with the increase in weight fraction of Copper nanobud particles was due to the interfacial polarization. When the prepared composite material samples are placed in an electric field, the charged particles of the nanobuds interact with the applied field. Therefore, if the material is a conductor, then the free electrons transfer to the nearby positive electrode. Besides, when no electric field exists, the free electrons remain within the material. If a material is nonconducting in another term dielectric, then the electrons are only locally displaced because they are bound to individual atoms. The local displacement polarizes the material. For the low weight fraction, when the frequency is increased to $10^{6} \mathrm{~Hz}$, the dielectric loss is approximately constant. This condition is attributed to the mechanisms of other types of polarization that occurs at high frequencies. These findings are in agreement with reported results in [35].

\section{Conclusion}

The effect of weight fraction and frequency on dielectric properties of polymethylmethacrylate filled with Copper nanobud particles has been investigated in this paper. The prepared Copper nanobuds were characterized by $\mathrm{UV}$-Vis spectroscopy and XRD. The results show that the Copper nanoparticles have bud shapes, and their average particle size obtained from the XRD study is $53.78 \mathrm{~nm}$. The presence of UV-Vis absorption peak at $574.8 \mathrm{~nm}$ is attributed to the formation of Copper nanobuds. When frequency increases, the dielectric loss factor ( $\left.\epsilon^{\prime \prime}\right)$ and dielectric constant $\left(\epsilon^{\prime}\right)$ are diminished. By contrast, the dielectric loss factor and dielectric constant increase as the weight fraction increases. Besides, the dissipation factor $(\tan \delta$ ) has a similar behavior to that of dielectric constant and dielectric loss factors in terms of frequency and weight fraction of Copper nanobud particles. The dielectric parametric investigations are advantageous to realize their application limitations not only for electronic apllications but also for practicable medical uses. Additional future work can be done by investigating particle size dependent study on the performance of medical PMMA polymer filled with copper nanobud particles.

\section{References}

1. N. Awaya, et al., Evaluation of a Copper metallization process and the electrical characteristics of Copper-interconnected quarter-micron CMOS. IEEE Transactions on Electron Devices, 1996. 43: p. 1206-1212.

2. R.J. Gutmann, Advanced silicon IC interconnect technology and design: present|ntrends and RF wireless implications. 
IEEE Transactions on Microwave Theory and Techniques, 1999. 47: p. 667-674.

3. J.H. Golden, C.J. Hawker, and P.S. Ho, Designing porous low- $k$ dielectrics. Semiconductor International, 2001. 24: p. 7988.

Li, M.M., et al., Dielectric properties of electrospun titanium compound/polymer composite nanofibres. Chinese Physics B, 2010. 19.

5. N.Aoi, Novel Porous Films Having Low Dielectric Constants Synthesized by Liquid Phase Silylation of Spin-On Glass Sol for Intermetal Dielectrics. Japanese Journal of Applied Physics, 1997. 36: p. 1355-1359.

6. Wu, W.-1., et al., Properties of nanoporous silica thin films determined by high-resolution $x$ ray reflectivity and small-angle neutron scattering. Journal of Applied Physics, 2000. 87: p. 1193.

7. Jo. M.H., et al., SiO2aerogel film as a novel intermetal dielectric. Journal of Applied Physics, 1997. 82: p. 1299-1304.

8. A.Kelly, Concise encyclopedia of composite materials. 2012.

9. A. Mortensen, Concise encyclopedia of composite materials. 2006.

10. M. Xanthos, Functional fillers for plastics. 2010.

11. J . Delmonte, Metal/polymer composites. 2013.

12. J.C . D.R .Denison, Barbour, and J.H. Burkhart, Low dielectric constant, fluorine-doped $\mathrm{SiO}_{2}$ for intermetal dielectric. Journal of Vacuum Science \& Technology A: Vacuum, Surfaces, and Films, 1996. 14: p. 1124-1126.

13. M .Tada., et al., $\mathrm{Cu}$ dual damascene interconnects in porous organosilica film with organic hard-mask and etch-stop layers for 70 nm-node ULSIs, in Interconnect Technology Conference, 2002. Proceedings of the IEEE 2002 International. 2002. p. 12-14.

14. M., A.Akram, Javed, and T.Z. Rizvi, Dielectric properties of industrial polymer composite materials. Turkish Journal of Physics, 2006. 29: p. 355-362.

15. K .Majdi, and H. Fadhal, Electrical conduction of PMMA and the effect of graphite addition. Univ. of Basrah, Iraqi of Polymers, 1997. 1(1): p. 15-20.

16. C.Basavaraja., et al., Preparation, characterization and low-frequency ac conduction of polypyrrole-lead titanate composites. Bulletin of the Korean Chemical Society, 2007. 28: p. 1104-1108.

17. Z .Al-Ramadhan, Effect of Nickel salt on electrical properties of polymethylmethacrylate. Journal of College of Education, 2008: p. 321399.

18. S .Bhattacharya,., R.P. Tandon, and V.K. Sachdev, Electrical conduction of graphite filled high density polyethylene composites; experiment and theory. Journal of materials science, 2009. 44: p. 2430-2433.

19. B.Hussien, M. Abdul-Muhsin, and A. Hashim, Study of some electrical properties for PMMA$\mathrm{TiO}_{2}$ composites. Atti Della Fondazione Giorgio Ronchi Anno LXVI N. 1, 2010: p. 45.

20. B.Hussien, The $D C$ and $A C$ electrical properties of (PMMA-Al2O3) composites. Eur $\mathrm{J}$ Sci Res, 2011. 52: p. 236-242.

21. S.H .Mohammad., The Effect of Different Water Solution on the Electrical Conductivity of Polyester Reinforced With Waste Aluminum. AlNahrain Journal for Engineering Sciences, 2015. 18: p. 61-65.

22. A.W .Watan,. and S.H. Aleabi, Studying Some Effective Parameter on The Dielectric Properties of Composite Materials Containing of Un Saturated Polyester Resin. Engineering and Technology Journal, 2015. 33: p. 83-90.

23. M.Sampath,., et al., Green synthesis of novel jasmine bud-shaped Copper nanoparticles. Journal of Nanotechnology, 2014. 2014.

24. S.Tan,.., et al., Facile fabrication of Coppersupported ordered mesoporous carbon for antibacterial behavior. Materials Letters, 2010. 64: p. 2163-2166.

25. D.B.Pedersen., S. Wang, and S.H. Liang, Charge-Transfer-Driven Diffusion Processes in $\mathrm{Cu}$ @Cu-Oxide Core-Shell Nanoparticles: Oxidation of $3.0 \pm 0.3 \mathrm{~nm}$ Diameter Copper Nanoparticles. The Journal of Physical Chemistry C, 2008. 112: p. 8819-8826.

26. T. M.A.Ghodselahi,. Vesaghi, and A. Shafiekhani, Study of surface plasmon resonance of $\mathrm{Cu} @ \mathrm{Cu} 2 \mathrm{O}$ core-shell nanoparticles by Mie theory. Journal of Physics D: Applied Physics, 2008. 42(1): p. 015308.

27. L .Balogh. and D.A. Tomalia, Poly (amidoamine) dendrimer-templated nanocomposites. 1. Synthesis of zerovalent Copper nanoclusters. Journal of the American Chemical Society, 1998. 120: p. 7355-7356.

28. R.M. Crooks., et al., Dendrimer-encapsulated metal nanoparticles: synthesis, characterization, and applications to catalysis. Accounts of chemical research, 2001. 34: p. 181-190.

29. R. Pethig, Dielectric properties of biological materials: biophysical and medical applications. IEEE Transanctions of Electrical Insulators. 1984;EI-19:;453-474.

30. G. Anand, A. Lowe, A. Al-Jumaily, Tissue phantoms to mimic the dielectric properties of human forearm section for multi-frequency bioimpedance analysis at low frequencies. Materials Science \& Engineering C (2018), https://doi.org/10.1016/j.msec.2018.11.080. 
31. Gwinstek LCR8000G user manual, LCR-8000G Series. www.gwinstek.com.

32. M. Kutz, Handbook of materials selection. 2002.

33. A .Moores, and F. Goettmann, The plasmon band in noble metal nanoparticles: an introduction to theory and applications. New Journal of Chemistry, 2006. 30: p. 1121-1132.

34. A.T .Shah., et al., In situ synthesis of Copper nanoparticles on SBA-16 silica spheres. Arabian Journal of Chemistry, 2016. 9(4): p. 537-541.

35. M.N. A. Abd Alameer, S. H. Mohammad, Studying the Effect of Aluminum Powder Addition on Dielectric Behavior of Polyester Composite Materials, Eng. \& Tech. Journal, 2013, 31(7): 1388-1394. 